EPJ Web of Conferences 60, 18009 (2013)

DOI: $10.1051 /$ epjconf $/ 20136018009$

(C) Owned by the authors, published by EDP Sciences, 2013

\title{
SUSY searches for EWK production of Gauginos and Sleptons at the LHC by CMS
}

\author{
Marc Dünser ${ }^{1, a}$ \\ ${ }^{1}$ ETH Zürich
}

\begin{abstract}
We search for signs of new physics in events with multi-leptonic final states in $9.2 \mathrm{fb}^{-1}$ of protonproton collisions at $\sqrt{s}=8 \mathrm{TeV}$ with the CMS experiment [1]. The final states under study comprise exactly three leptons, four leptons, a pair of same-sign leptons, two opposite-sign-same-flavor leptons plus jets, and two opposite- sign leptons which have a mass outside the Z-boson decay window. Observations agree well with the expectations from the standard model. The obtained results are then interpreted as limits on the direct electroweak SUSY production of charginos, neutralinos and sleptons.
\end{abstract}

\section{Introduction}

Early searches for SUSY at the LHC focussed on strong production of gulinos and squarks, which usually feature events with high hadronic activity. In the present search, however, we focus on searches motivated by the direct production of charginos, neutralinos, and sleptons, as such production modes would have eluded most of the early searches. We concentrate on leptonic final states with either exactly three leptons, four leptons, same-sign dileptons, opposite-sign-same-flavor leptons with jets, and opposite-sign leptons outside of the Z-boson decay window. We interpret our results in terms of simplified model spectra (SMS) [2].

\section{Final states}

\section{Three leptons}

We require three well-isolated leptons of which one can be a hadronically decaying $\tau$-lepton in any flavor and sign combination. A veto on jets identified as originating from bottom quarks is implemented in order to suppress contributions from $t \bar{t}$ production and a moderate requirement on missing transverse momentum $\left(E_{\mathrm{T}}^{\text {miss. }}\right)$ are employed to further suppress the background. The search is then binned in four bins of $E_{\mathrm{T}}^{\text {miss. }}$ of $50 \mathrm{GeV}$ each, three bins in the transverse mass $\left(M_{\mathrm{T}}\right)$ of the remaining lepton if an opposite-sign-same-flavor (OSSF) pair is present. For events without and OSSF pair, the pair closest to the Zmass is used for $M_{\ell \ell}$ and the remaining for the calculation of $M_{\mathrm{T}}$. Another three bins in $M_{\ell \ell}$ are introduced for events with an OSSF pair in the event. The observations agree well with the predictions for the backgrounds.

\section{Same-sign dileptons}

In the same-sign dilepton search we require two very isolated leptons (e or $\mu$ ) with equal charge and veto any third lepton that would pass the three lepton analysis selection in order to be statistically exclusive for combining the results. This channel is specifically interesting for any compressed spectra if one of the leptons is lost or out of acceptance. We have two bins for our search, one with a tight requirement of $E_{\mathrm{T}}^{\text {miss. }}>200 \mathrm{GeV}$ and another with a relaxed requirement on $E_{\mathrm{T}}^{\text {miss. }}$ of 120 to $200 \mathrm{GeV}$ where we additionally introduce a veto on jets identified as stemming from a b-quark and an upper bound on the number of jets in the event of two. Also in this channel, we do not observe any excess of data over the expectationd from the standard model.

\section{Four leptons}

The lepton selection in the four-lepton search is the same as in the three-lepton search, with again maximally one hadronically decaying $\tau$-lepton. We require the presence of at least one ee or $\mu \mu$ pair within $15 \mathrm{GeV}$ of the Zboson mass. Expectations from the standard model are well within in the observations.

\section{Opposite-sign-same-flavor leptons with jets}

For this search, we require one OSSF pair which has an invariant mass within $15 \mathrm{GeV}$ of the Z-boson mass (on-Z). Additionally, we require at least two jets recoiling against the leptonic sytem, while also implementing a veto on btagged jets. A moderate requirement on $E_{\mathrm{T}}^{\text {miss. }}$ of $50 \mathrm{GeV}$ is used to suppress the $\mathrm{Z}+$ jets background. We bin the analysis in a total of 5 search regions of $E_{\mathrm{T}}^{\text {miss. }}$, in all of

\footnotetext{
a e-mail: marc.dunser@cern.ch
} 
which we see good agreement between the observed data and the expectations.

\section{Non-resonant opposite-sign dileptons}

Additional to on-Z opposite sign leptons, we also search in the non-resonant opposite-sign channel. We require an invariant mass outside of the Z-mass window of $\pm 15 \mathrm{GeV}$ for the same-flavor case, and perform our search in a variable named $M_{\mathrm{CT} \perp}$ which features a strong suppression of the standard model background [3]. Standard model processes that feature two W-bosons in the final state have an endpoint in $M_{\mathrm{CT} \perp}$ at the $\mathrm{W}$-boson mass, while this does not hold for potential SUSY scenarios. We derive templates for the shape of $M_{\mathrm{CT} \perp}$ from data and simulation and fit the shape at low values of the variable while extracting our results from the tail of the distribution. The observed number of events agrees well with the predictions.

\section{Interpretations}

We interpret the aforementioned results in a number of different SMS, according to the sensitivity of each final state to the objects in respective SUSY-decays.

\subsection{Chargino-Neutralino production}

We consider production of one chargino $\left(\widetilde{\chi}_{1}^{ \pm}\right)$and one neutralino $\left(\widetilde{\chi}_{2}^{0}\right)$, each decaying fully leptonically via sleptons $(\widetilde{\ell})$. The intermediate slepton masses are defined by a parameter $\mathrm{x}$ in the following way:

$$
m_{\tilde{\ell}}=m_{\widetilde{\chi}_{1}^{0}}+x\left(m_{\widetilde{\chi}_{1}^{ \pm}}-m_{\widetilde{\chi}_{1}^{0}}\right),
$$

where $0<x<1$. Thus, this parameter defines the level of spectrum compression. For a value of $x=0.5$, only the three-lepton results are used for the interpretation. Two examples can be seen in Fig. 1 for a flavor democratic scenario of the chargino decay (left) and a tau-enriched scenario (right), where the chargino only decays into stau and neutrino. For more extreme values of $\mathrm{x}$, the inclusion of the same-sign dilepton results shows a considerable increase in sensitivity compared to the three-lepton final state alone, especially along the diagonal, where a third lepton might be lost due to the acceptance lepton selection. Figure 2 shows exclusion curves and upper limits on the cross-section times branching fraction for the flavor-democratic scenario (left) and the tau-enriched scenario (right), where the value of $x$ has been shifted to 0.05 .

We consider also decays of the chargino and neutralino via on-shell vector bosons, for which the upper limits and exclusions can be seen in Fig. 3. Both the three-lepton analysis as well as the OSSF+jets analysis were used to derive these exclusions.

\subsection{Chargino-, and Slepton-pair production}

For the pair-production of charginos and sleptons (only $\tilde{e}$ and $\tilde{\mu}$ ), we use the non-resonant opposite-sign dilepton

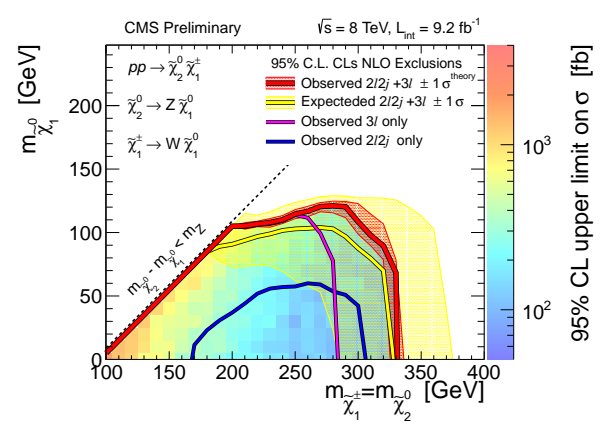

Figure 3. Upper limits and exclusions in chargino-neutralino production with the decay through vector bosons.

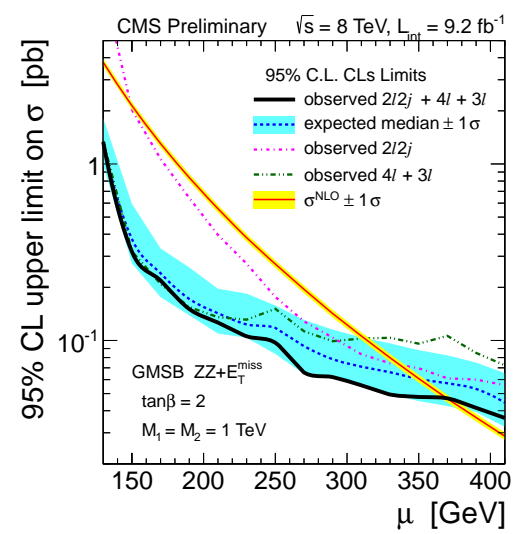

Figure 5. Exclusions for neutralino-pair production in a GMSB motivated scenario.

search for the interpretations. The exclusions can be found in Fig. 4 for the chargino-pair production (left) and the slepton pair-production (right). For the chargino-decay, we only consider intermediate sleptons where $\mathrm{x}$ is set to 0.5 .

\subsection{Neutralino-pair production}

We consider neutralino-pair production and decay in a gauge-mediated SUSY breaking scenario (GMSB) via two Z-bosons to the gravitino, the almost massless lightest supersymmetric particle in this model. Exclusions in terms of the gaugino mass parameter $\mu$ (where $m_{\widetilde{\chi}_{1}^{0}} \approx m_{\widetilde{\chi}_{1}^{ \pm}} \approx$ $m_{\widetilde{\chi}_{2}^{0}} \approx \mu$ ) are displayed in Fig. 5. The four-lepton final state in combination with the OSSF+jets final state were used for this particular model.

\section{Conclusions}

We performed searches for direct electroweak production of SUSY with the CMS experiment on a dataset of $9.2 \mathrm{fb}^{-1}$ of $8 \mathrm{TeV}$ p-p collisions. In the light of good agreement between observations and expectations, we proceed to set limits in the context of simplified model spectra. We are able to probe masses of charginos and neutralinos of up to $650 \mathrm{GeV}$ and slepton masses of up to $275 \mathrm{GeV}$. 

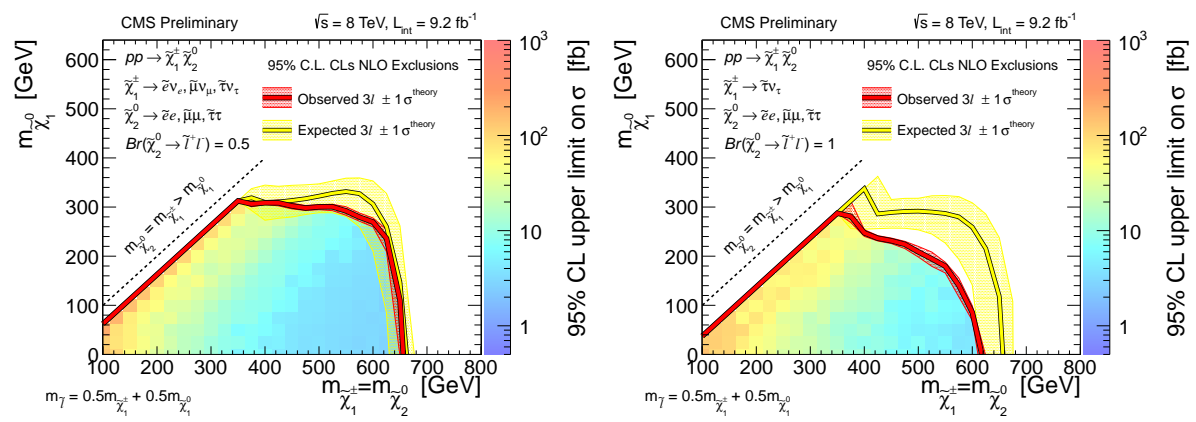

Figure 1. Upper limits on the cross-section times branching fraction as well as exclusion curves for chargino-neutralino production where $\mathrm{x}=0.5$.
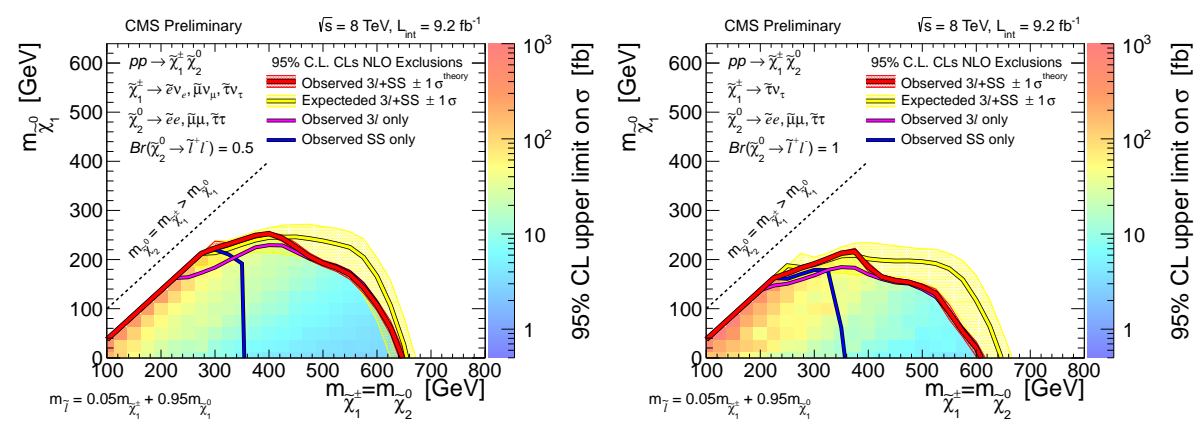

Figure 2. Exclusion curves for chargino-neutralino production where $\mathrm{x}=0.05$.
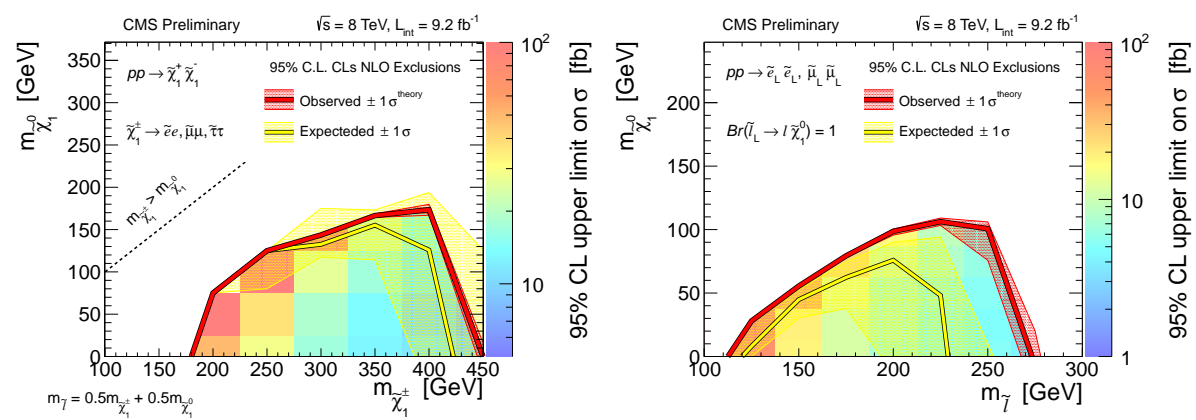

Figure 4. Exclusion curves for chargino-pair production (left) and slepton-pair production (right).

\section{References}

[1] CMS Collaboration, "The CMS experiment at the CERN LHC", JINST 3, S08004 (2008) doi:10.1088/1748-0221/3/08/S08004

[2] CMS Collaboration, "Search for electroweak production of charginos, neutralinos, and sleptons using lep- tonic final states in pp collisions at $\sqrt{s}=8 \mathrm{TeV}$ ", CMS Physics Analysis Summary CMS-SUS-12-022, 2012

[3] K. T. Matchev and M. Park, "A General method for determining the masses of semi-invisibly decaying particles at hadron colliders", Phys. Rev. Lett. 107 061801 (2011) doi:10.1103/PhysRevLett.107.061801, arXiv:0910.1584. 